\title{
Aluminum Nanoparticles as Fiducials for Nanoscale Temperature Measurements
}

\author{
Matthew Mecklenburg ${ }^{1}$, Brian Zutter ${ }^{2}$, and B. C. Regan ${ }^{2}$ \\ ${ }^{1 .}$ Center for Electron Microscopy and Microanalysis, University of Southern California, Los Angeles, \\ CA, USA \\ 2. Department of Physics and Astronomy \& California NanoSystems Institute, University of California, \\ Los Angeles, CA, USA
}

In plasmon energy expansion thermometry (PEET), electron energy-loss spectroscopy (EELS) is used to determine the plasmon energy in a suitable thermometric material [1]. Since the plasmon energy scales with the material's density, temperature changes can be determined using knowledge of the material's thermal expansion coefficient. Many in situ transmission electron microscopy experiments could benefit from having thermometric fiducials, i.e. localized thermometers within the field of view. Nanoparticles of aluminum, with their narrow bulk plasmon resonance and large thermal expansion coefficient $[2,3]$, are particularly well suited to act as such fiducial thermometers.

Using an FEI NanoEx-i/v sample holder we applied PEET to aluminum nanoparticles held at temperatures ranging from $22^{\circ} \mathrm{C}$ (room temperature) to $500^{\circ} \mathrm{C}$, as measured by the holder's built-in resistance thermometer. A plasmon energy map was generated from a spectrum image acquired at each temperature through the procedure described in Ref [1]. Plasmon energy maps acquired at the extremes of the temperature range are shown in Figures 1(A-B), as are the histograms of the plasmon energies. Two Gaussians are used to model the plasmon energy distributions because the edge of the nanoparticle has a plasmon energy 20-25 meV larger than the center value. This difference between the bulk and edge plasmon energies, which indicates that the surface is $\sim 0.1 \%$ denser than the bulk, remains constant as a function of temperature. All the nanoparticles we examined had a similar shift. A PEET-determined temperature map made from the two plasmon maps is shown in Figure 1(C). Because the edge-to-center plasmon shift is temperature-independent we find the nanoparticle to be isothermal, as expected.

In a typical experimental run [Figure 2] we acquired two room temperature plasmon maps, then jumped to $500^{\circ} \mathrm{C}$, and then ramped in $50^{\circ} \mathrm{C}$ steps back to room temperature [Figure 2(A)]. The relative shift in the plasmon energy, as determined by the histogram fits, was compared with the expected relative change due to thermal expansion [Figure 2(B)]. Similarly, the PEET temperature is computed using the known thermal expansion coefficient and compared to the calibrated holder temperature [Figure 2(C)]. Over the entire range of temperatures the PEET temperature, measured to a precision of $8 \%$, agrees with the sample holder's calibrated temperature to within $4 \%$, which is better than the $5 \%$ accuracy of the holder calibration. This good agreement indicates that aluminum nanoparticles can be used as thermometric fiducials over this temperature range.

\section{References:}

[1] M Mecklenburg et al, Science 347 (2015), p. 629-632.

[2] G Meyer, Zeitschrift fur Physik 148 (1957), p. 61-71.

[3] A J C Wilson, Proceedings of the Physical Society 54 (1941), p. 235-244.

[4] This work was supported in part by FAME, one of six centers of STARnet, a Semiconductor Research Corporation program sponsored by MARCO and DARPA. The data were acquired at the FEI NanoPort (Oregon, USA) using a FEI Titan Themis 80-200. 

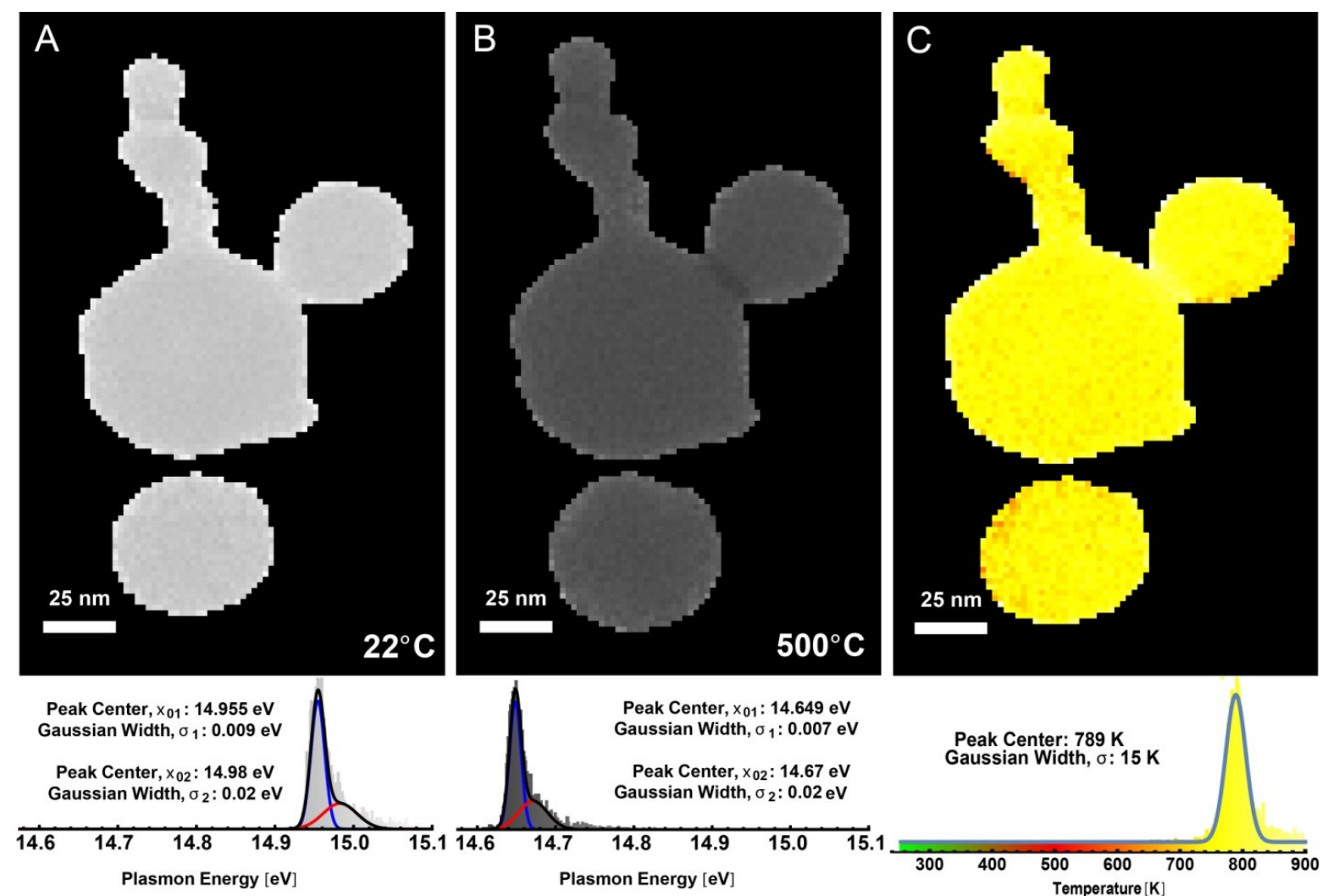

Figure 1. (A, B) Aluminum plasmon energy maps (116 pixels tall by 73 pixels wide, 2 nm pixel size) extracted from spectrum images acquired at $22^{\circ} \mathrm{C}$ and $500^{\circ} \mathrm{C}$, respectively. The plasmon energy is taken to be the energy difference between the zero-loss and first plasmon resonance peaks, as determined by Gaussian fits. Histograms of the plasmon energies are shown below the maps, as are the fit parameters to the two Gaussians used to fit the histogram. (C) Temperature map made from the (A, B) maps by applying the PEET technique. The standard deviation of temperatures in the map is $15 \mathrm{~K}$.
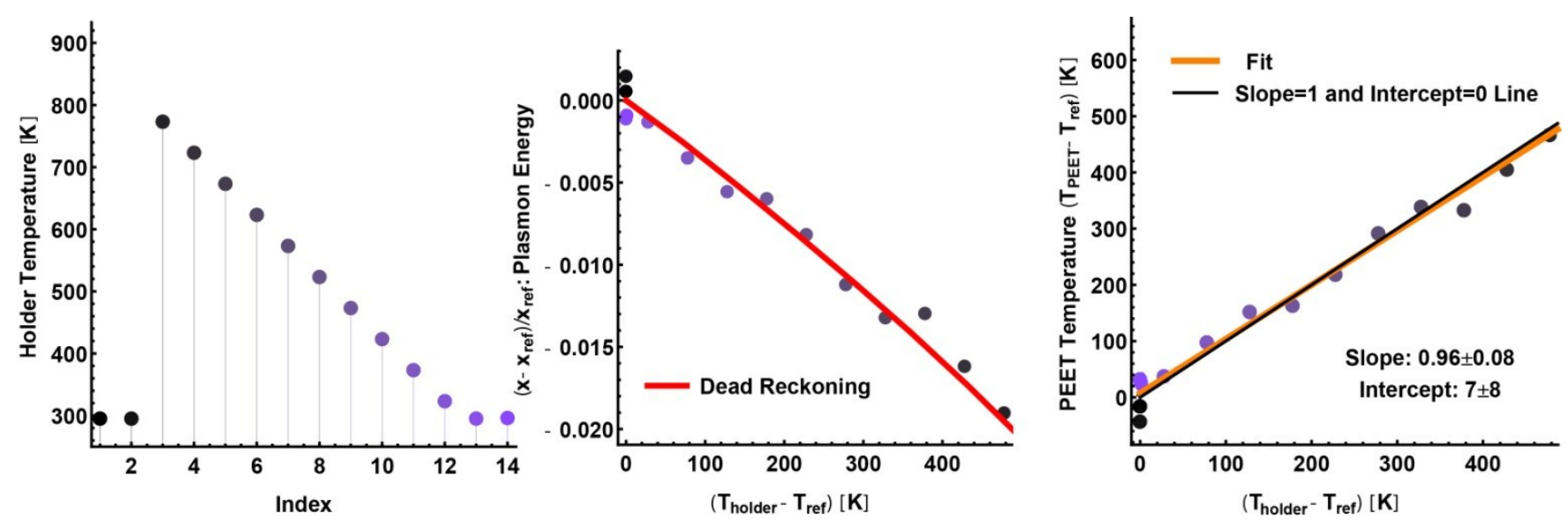

Figure 2. The calibrated holder temperature (left) and relative shift in the plasmon energy in the aluminum nanoparticles (center). The dead reckoning line is a comparison to the expected value from the known thermal expansion coefficient in aluminum ( $\mathrm{T}_{\mathrm{ref}}$ is the average of the holder temperatures at points 1, 2, 13, and 14). The PEET-determined temperature vs. the calibrated heater temperature (right). 